\section{Differential effects of familiarity on judgments of sameness and difference*}

\author{
HOWARD EGETH $\dagger$ and DAVID BLECKER $\dagger \dagger$ \\ The Johns Hopkins University, Baltimore, Maryland 21218
}

Ss indicated whether pairs of simultaneously presented objects were "same" or "different." In Experiments 1, 2, and 3 the stimuli were pairs of letters, and familiarity was manipulated by showing the letters in either an upright or an upside-down orientation. In Experiments 4 and 5 the stimuli were pairs of trigrams, and familiarity was manipulated either by rotation or by selection according to rated meaningfulness. Analysis of reaction times indicated that familiar pairs were responded to more quickly than were unfamiliar pairs; however, this was true only for "same" judgments, not for "different" judgments. In addition, Experiment 3 indicated that familiarity influenced discrimination accuracy under conditions of tachistoscopic exposure. Finally, in Experiment 6 an effort was made to disentangle the effects of meaningfulness from the effects of pronounceability. The present results stand in contrast to previous research using perceptual comparison tasks, since the earlier work failed to indicate any effect of familiarity.

Is the perception of stimuli influenced by their familiarity? This fundamental question has engaged the interest of experimental psychologists for many years. As a result of this keen interest there now exists a large body of literature which indicates that familiar stimuli are perceived more readily or more accurately than are unfamiliar stimuli (e.g., Howes \& Solomon, 1951; Wheeler, 1970). However, there also exists a substantial body of evidence that suggests either that perceptibility is unrelated to familiarity (e.g., Robinson, Brown, \& Hayes, 1964) or that familiarity influences performance through the mediation of nonperceptual factors such as response bias (e.g., Goldiamond \& Hawkins, 1958).

Some of the strongest evidence that familiarity does not affect perceptibility comes from studies of perceptual comparison (also called matching or discrimination). In these experiments Ss were presented with pairs of stimuli and asked to indicate whether the items were the same or different (Hochberg, 1968: Posner \& Mitchell, 1967; Robinson, Brown, \& Hayes, 1964). There are several reasons for paying close attention to the

*This rescarch was supported by a grant from the Office of Education of the U.S. Department of Health, Education and Welfare. The authors would like to thank Netson Bingham and Deborath Hall for their help in running $S_{s}$ and analyzing data. This paper has benefited from suggestions made by Donald Bamber. Julian Hochberg, Howard Hock, Robert Pachella, and Edward Smith. Part of this rescarch was reported at the 1969 meetings of the Psychonomic Society.

$\pitchfork$ Requests for reprints should be sent to Howard Egeth, Department of Psychology. Johns Hopkins University, Baltimore, Maryland 21218.

+ Now at Tufts University Medical School. results of perceptual comparison experiments. First, the binary response of "same" or "different" eliminates the response bias artifact that plagued earlier studies in which Ss had to verbalize the presented material. Second, since most research on familiarity effects has used recognition or identification tasks, perceptual comparison provides a useful converging operation. Third, the task of immediate perceptual comparison seems to be at a low enough level in the hierarchy of information processing functions (Egeth. Blecker, \& Kamlet, 1969) that it seems intuitively to be a good vehicle for studying perception. Despite its virtues, it should be pointed out that the comparison method is only one wisy, and a fairly restrictive one at that of studying perception. Therefore, the results of such research should be generalized to indicants of other aspects of perception only with considerable caution.

The present investigation was undertaken because none of the three perceptual matching studies cited above seemed quite convincing. However, even if that work were completely convincing, the conclusion that familiarity does not affect perception is of sufficient theoretical interest to warrant further research.

Posner and Mitchell drew their conclusions about familiarity from the comparison of matching data from conditions in which pairs of unfamiliar "Gibson figures" were the stimuli and data in which ordinary letter pairs were the stimuli. However, in these experiments the Ss were instructed to indicate whether of not the stimuli had the same name (e.g., a pair consisting of " $A$ " and " $a$ " would be considered "same"). Since matching was on the basis of conceptual rather than perceptual identity, the data do not bear directly on the issue of whether familiarity influences perceptual processes.

Hochberg reported a study in which Ss were presented with pairs of long letter strings printed side by side in vertical columns. In the condition of high familiarity, the letter strings were, essentially, English words. Two different kinds of unfamiliar stimuli were employed. In one the strings were meaningless combinations of letters, and in the other the letters of the words were mirror images of ordinary letters. When the two letter strings were presented close together, the times taken to indicate whether the elements were the same or different were equivalent for the familiar pairs and the two kinds of unfamiliar pairs. Interestingly, when the letter strings were far apart on the page, and thus required greater memory capacity for correct matching, a familiarity effect was obtained. The research design was probably not sensitive enough to detect fairly small differences in judgment time. ${ }^{1}$ In one version of the experiment, Ss were given as many $1.35 \mathrm{sec}$ exposures of the stimuli as they desired before making their decisions. The dependent variable was the number of such presentations required before they responded (typically one, two, or three). With pairs of long letter strings, counting the number of glances required to reach a decision is useful to the extent that familiarity influences the size of the unit the Ss can handle in making comparisons. However, as important as that issue may be in its own right, the number of looks does not speak directly to the question of how perceptible stimuli are, cspecially when the size of the stimuli may be less than one "look-full." For this purpose a continuous measure of judgment time would seem preferable.

In the study by Robinson et al pairs of letters were presented tachistoscopically with the letters in either their ordinary orientation (familiar) or as mirror images (untamiliar). The "same"-"different" task was used in conjunction with an ascending method of limits, and discrimination thresholds were obtained. These thresholds proved to be unrelated to the familiarity of the stimulus elements being compared. Unfortunately, since the details of the experimental procedure were not presented in this paper, it is impossible to evaluate the results of this study.

The present series of experiments may be viewed as an effort to replicate the essential features of the previous matching experiments, hopefully with somewhat more suitable experimental designs. As will become evident, the data provide the unexpected dividend of casting some light 
on the nature of judgments of sameness and difference.

\section{EXPERIMENT 1}

Method

Subjects. Four male and four female volunteers from the Johns Hopkins summer session served individually in an experimental session that lasted for about 20 min. No $S$ served in more than one of the experiments in this series. The $S s$ received $\$ 1.50$ for participating.

Stimuli. Pairs of 30-point Prestype uppercase letters (Folio Medium Extended) were prepared on white cards. The letters were arranged next to one another on a horizontal line and were separated by $11 \mathrm{~mm}$ center-to-center. They were equidistant from a central fixation point and appeared at a viewing distance of $84 \mathrm{~cm}$. The stimulus series was made by making two identical decks of 36 cards each; one of these decks was then rotated by $180 \mathrm{deg}$, and the 72 cards were put in a random order which was the same for all Ss. Thus, on half of the 72 trials the two letters appeared in a normal (familiar) orientation, and on half of the trials the letters were both upside-down (unfamiliar). Furthermore, on half of the trials the two letters in a pair were different from one another and on the other half the same. Letters such as $O$ and $X$, which, in that typeface, looked the same in both orientations, were avoided in the preparation of stimuli.

Procedure. The $\mathrm{S}$ sat at a Scientific Prototype two-channel tachistoscope. Between trials a small fixation point indicated the center of the visual field. Each trial consisted of the presentation of a stimulus card for $1.0 \mathrm{sec}$. The Ss were instructed to indicate whether the two letters on the card were the same or different by pressing one of two appropriately labeled keys. Half of the Ss pressed the "same" key with the thumb of the preferred hand and the "different" key with the thumb of the other hand, while this mapping was reversed for the other half of the Ss.

Response latency was measured from the onset of the stimulus to the depression of the response key. Errors were also noted and reported to the $S$, although no feedback was provided about response speed.

The Ss were instructed to "... work accurately, and within that limitation as quickly as possible." They were told in advance the number of trials they would receive and that on about half of the trials the stimuli would be the same and on half different. They were urged to make the "same"."different" judgment a visual one and to avoid naming the stimuli. Six practice trials were given.
Table 1

Mean Reaction Times (Msec), Experiment 1

\begin{tabular}{lccc}
\hline & Unfamiliar & Familiar & $\Delta^{\mathrm{a}}$ \\
\hline Same & 549 & 500 & 49 \\
Different & 555 & 560 & -5 \\
\hline
\end{tabular}

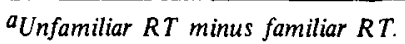

\section{Results}

Mean reaction times (RTs) for the four experimental conditions are presented in Table 1. The times from trials on which Ss made errors were excluded from consideration. Since error rates were only about $2 \%$ in the present series of experiments, the conclusions are probably unaffected by eliminating error trials (Egeth \& Smith, 1967).

An analysis of variance carried out on these data indicated that the main effects of familiarity and response as well as their interaction were significant. For familiarity, $F(1,6)=11.63, p<.025$; for "same" vs "different," $F(1,6)=41.36$, $\mathrm{p}<.001$; and for the interaction, $F(1,6)=8.68, p<.05$. It is important to note the form of the interaction. For "different" judgments there is not a significant difference between the speed of response to familiar and unfamiliar pairs, but for "same" judgments the familiar orientation resulted in substantially faster responses than did the unfamiliar orientation.

\section{EXPERIMENT 2}

The nature of the interaction between familiarity and response observed in Experiment 1 suggested that judgments of sameness and difference may be mediated, at least in part, by independent processes. It seemed worthwhile to attempt to extend the generality of this conclusion by changing the nature of the task. To this end, the two-response task of Experiment 1 was replaced by a one-response task (a Donders Type c reaction) in all of the following reaction-time experiments. Half of the Ss were instructed to respond by pressing a key with the thumb of the preferred hand when the stimuli were "same," but to refrain from responding when the stimuli were "different." The other half of the Ss were instructed to respond by a keypress with the thumb of the preferred hand when the stimuli were "different" but to refrain from responding when the stimuli were "same."

Table 2

Mean Reaction Times (Msec), Experiment 2

\begin{tabular}{lccc}
\hline & Unfamiliar & Familiar & $\Delta^{\mathrm{a}}$ \\
\hline Same & 461 & 410 & 51 \\
Different & 540 & 529 & 11 \\
\hline
\end{tabular}

a Unfamiliar $R T$ minus familiar $R T$.
The one-response task was used with 16 Ss in Experiment 2. In all other respects the study was identical to the previous one.

\section{Results}

Mean RTs for the four conditions appear in Table 2. As in the previous experiment both main effects as well as thejr interaction were significant. For familiarity, $F(1,14)=15.93, p<.005$; for "same" vs "different," $F(1,14)=5,10$, $p<.05 ;$ and for the interaction of Familiarity by Response, $\mathrm{F}(1,14)=6.96$, $p<.025$. The pattern of results is similar to that obtained in the first experiment; a small and statistically nonsignificant difference between RTs to familiar and unfamiliar stimuli when they were different, but a large difference when they were the same. It is also interesting to note that the change in procedure from a two-response task to a one-response task reduced all RTs, as can be seen by examining the comparable cells of Tables 1 and 2. Furthermore, this procedural changc had a much greater effect on "same" latencies than on "different" latencies. This observation is consistent with the suspicion that there may be independent mechanisms for the detection of sameness and difference.

\section{EXPERIMENT 3}

Having obtained clear-cut results in two reaction-time experiments, it seemed worthwhile to explore familiarity effects when the stimuli were available for comparison for only a brief time. The exposure durations used in this study were intended to approximate the brief exposures used in the early studies of familiarity effects that used recognition threshold as the dependent variable (e.g., Howes \& Solomon, 1951), as well as the study by Robinson et al (1964) in which discrimination thresholds were determined. However, to avoid violating the canons of current psychophysical theory, the dependent variable was not threshold but a nonparametric analog of the $d^{\prime}$ measure of signal detection theory (e.g., Pollack, Norman, \& Galanter, 1964). We shall use the notation $A_{\mathrm{g}}$ for this measure.

\section{Method}

Subjects. Twenty paid volunteers served, 8 in Part 1 and 12 in Part 2.

Stimuli. Additional cards, similar to those used in Experiments 1 and 2 were prepared and randomly mixed with the originals, making a total of 144 cards. Each of the cards was shown once, in a random order that was the same for all Ss.

Procedure. The Ss were instructed to indicate vocally whether the flashed letters were the same or different. In addition, they were asked to rate their confidence in 
Table 3

$A_{g}$ for Familiar and Unfamiliar Pairs, Experiment 3

\begin{tabular}{|c|c|c|c|c|c|}
\hline \multicolumn{3}{|c|}{ Part $1(60 \mathrm{Msec})$} & \multicolumn{3}{|c|}{ Part $2(70 \mathrm{Msec})$} \\
\hline$S$ & Familiar & Unfamiliar & $\mathrm{S}$ & Familiar & Unfamilia \\
\hline 1 & .85 & .80 & 9 & .83 & .76 \\
\hline 2 & .80 & .71 & 10 & .91 & .67 \\
\hline 3 & .53 & .55 & 11 & .49 & .52 \\
\hline 4 & .51 & .54 & 12 & .83 & .80 \\
\hline 5 & .68 & .58 & 13 & .98 & .92 \\
\hline 6 & .67 & .74 & 14 & .92 & .88 \\
\hline 7 & .83 & .77 & 15 & .67 & .74 \\
\hline 8 & .52 & .58 & 16 & .89 & .86 \\
\hline & & & 17 & .74 & .66 \\
\hline & & & 18 & .66 & .59 \\
\hline & & & 19 & .80 & .78 \\
\hline & & & 20 & .93 & .95 \\
\hline
\end{tabular}

each of their decisions on a 6-point scale (sure same, half sure same, unsure same, unsure different, half sure different, sure different). What these confidence descriptions lacked in logical rigor they more than made up for in the ease with which Ss grasped their meanings.

In Part 1 each pair was shown once at a 60-msec exposure duration, and in Part 2 each pair was shown once for $70 \mathrm{msec}$. Visual noise, consisting of bits and pieces of letters, was present in the "blank" field of the tachistoscope both before and after the stimulus flash.

\section{Results}

Accuracy and confidence were analyzed together by computing, for each $\mathrm{S}, \mathrm{A}_{\mathrm{g}}$ scores for familiar pairs and unfamiliar pairs. These data appear in Table 3. Looking simply at the signs of the differences in $A_{g}$ between familiar and unfamiliar pairs, it is evident that Part 1 does not provide conclusive evidence of superior performance with familiar pairs. However, the three best Ss do show such advantage. It was precisely this observation that prompted us to run additional Ss with a somewhat longer duration in order to improve the general level of performance.

In analyzing the data from Part 2, the data of $\mathrm{S} 11$ were eliminated since his performance clearly failed to exceed chance. (There can be no familiarity effect with either perfect or chance performance.) of the remaining $11 \mathrm{Ss}$, 9 show a higher $\mathrm{A}_{\mathrm{g}}$ for familiar than for unfamiliar stimuli $(\mathrm{p}<.05)$. It was reassuring to find that the same conclusion followed from an analysis based simply upon percent correct. Thus, a familiarity effect is obtained under conditions of tachistoscopic discrimination.

Some weaknesses of this experiment deserve mention. First, the number of trials (144) was small. Obviously more reliable probability estimates would have been obtained with more trials. Second, since the visual noise pattern was constant throughout the experiment, it is conceivable that chance factors of placement made it somewhat more interfering with the unfamiliar pairs than with the familiar ones. But considering the overall consistency of these first three experiments it seems unlikely that either of these weaknesses was actually important

\section{EXPERIMENT 4}

It seemed important at this point to try to extend the generality of the investigation by using stimuli other than letter pairs and a familiarity manipulation other than letter rotation. CVC trigrams were selected because norms exist for evaluating factors such as their meaningfulness and association value, factors closely related to familiarity (cf. Underwood, 1966, pp. 466-467). The use of trigrams of varying degrees of meaningfuiness is also useful in that it is similar to Hochberg's (1968) use of words vs random letter strings.

\section{Method}

Subjects. Sixteen paid volunteers were used in the kind of single-response task described in Experiment 2. Half were instructed to respond "same" and half "different."

Stimuli. Pairs of trigrams were typed horizontally in lowercase letters, with one menber of the pair directly above the other. The trigrams were approximately $12 \mathrm{~mm}$ wide and between 4 and $6 \mathrm{~mm}$ tall. The center-to-center distance between the lines was about $8 \mathrm{~mm}$.

The "familiarity" manipulation was actually a manipulation in terms of meaningfulness as evaluated by Archer (1961). Trigrams rated 90 or higher were considered familiar, while trigrams 26 and below were considered to be unfamiliar. As in all of the present experiments, both of the elements of a pair were familiar or both were unfamiliar. There were 72 trials, halt "same" and half "different." As usual, half of each kind were familiar and half were unfamiliar. "Different" pairs differed in one and only one letter, the first, second and third positions being equally often the locus of a difference.

It is difficult to select trigrams according to meaningfulness without at the same time biasing the frequency of use of particular letters (e.g., " $q$ " tends 10 appear relatively frequently in trigrams rated low in meaningfumess and infrequently in trigrams rated high). To determine if this was an important consideration in the comparison task, half of the 72 trials comprised a specially prepared set of stimuli in which meaningfulness was manipulated while controlling the frequency of use of particular letters. This
Table 4

Mean Reaction Times (Msec), Experiment 4

\begin{tabular}{lccr} 
& Unfamiliar & Familiar & \multicolumn{1}{c}{$\Delta^{\mathrm{a}}$} \\
\hline Same & 750 & 642 & 108 \\
Different & 644 & 648 & -4 \\
\hline
\end{tabular}

$a_{U}$ familiar $R T$ minus familiar $R T$.

was accomplished by the use of trigrams that were rated high when the letters were in one order and low when they were in the reverse order. For example, wig has a rating above 90 and its reverse, giw, has a rating below 26. Thus, if we had constructed a "same" pair using wig, then we would have also constructed a "same" pair using giw. The results for the specially constructed set and the less controlled pairs werc essentially identical, and so this problem will not be discussed further in the present paper.

\section{Results}

Mean RTs appear in Table 4. The main effect of familiarity was significant, $F(1,14)=15.98, \quad p<.005$, as was the interaction of Familiarity by Type of Response, $F(1,14)=17.99, \quad p<.001$, However, the main effect of "same" vs "different" (a between-S variable) was not significant

Once again, a very substantial difference in "same" RT between familiar and unfamiliar stimuli is coupled with an insignificant difference for "different" judgments.

\section{EXPERIMENT 5}

Hochberg (1968) found no familiarity effect with pairs of vertically arranged words. This experiment was designed to check on the (unlikely) possibility that something peculiar to vertical oricntation might have caused his results.

\section{Method}

Subjects. Sixteen new Ss were used in the one-response task described previously, half set for "same" and half set for "different."

Stimuli. Trigrams like those used in the previous experiment were typed vertically (i.e., with one letter above another) but with the trigrams themselves side by side. Half of the cards were turned upside down and thus the familiarity manipulation consisted of upright vs upside-down trigrams. $^{2}$

\section{Results}

Mean RTs appear in Table 5. The two main effects and their interaction were significant. For familiarity, $\mathrm{F}(1,14)=4.90$, $\mathrm{p}<.05$; for "same" vs "different," $F(1,14)=17.81, p<.001$; and for the interaction, $F(1,14)=5.96, p<.05$. As in 
Table 5

Mean Reaction Times (Msec), Experiment 5

\begin{tabular}{lccc} 
& Unfamiliar & Familiar & $\Delta^{\mathrm{a}}$ \\
\hline Same & 1049 & 954 & 95 \\
Different & 731 & 735 & -4 \\
\hline
\end{tabular}

$a_{\text {Unfamiliar }} R T$ minus familiar $R T$.

the preceding RT experiments, a familairity effect on "same" trials was coupled with an absence of such an effect on "different" trials. This makes it unlikely that Hochberg's data can be attributed to the vertical orientation of his stimuli.

There is an important difference between the data of this trigram study and the data of the two experiments using letter pairs (i.e., 1 and 2). Here "same" judgments were significantly longer than "different" judgments, while in the earlier experiments the "different" judgments were the longer. The results of Experiment 4, another trigram experiment, are not clear-cut in this regard, although they seem more like Experiment 5 than like Experiments 1 and 2.

\section{EXPERIMENT 6}

In Experiment 4 it was pointed out that the familiarity manipulation was actually a manipulation of rated meaningfulness. But since meaningfulness is itself a complex attribute, it is interesting to speculate further about the interpretation of the obtained results. In particular, Gibson"s efforts to disentangle the effects on perception of meaningfulness and pronounceability may be taken as a model for such speculation (Gibson, Pick, Osser, \& Hammond, 1962; Gibson, Bishop, Schiff, \& Smith, 1964). In the latter experiment, recognition thresholds for trigrams were determined by means of the ascending method of limits. The data indicated that pronounceable trigrams (e.g., BIF) were recognized at shorter exposure durations than were meaningful combinations of the same letters that were not pronounceable as a unit (e.g., FBI). Indeed, the meaningful arrangements were recognized no more quickly than were meaningless, unpronounceable control items (e.g., BFI). This general method of generating stimuli was used in the present study.

\section{Method}

Subjects. Ten Ss were used in a one-response task. They were all instructed to respond only to "same" stimuli.

Stimuli. Horizontal trigrams were used, arranged one above another as in Experiment 4. They were typed in uppercase letters on an ordinary elite typewriter. The type was about half the size used for Experiments 4 and 5 , resulting in generally longer RTs. Thirty-two cards were prepared, 16 containing "same" pairs and 16 containing "different" pairs. The 32 cards were presented in a different random order to each $S$. Of the 16 "same" pairs, 4 were fillers that did not enter into the analysis. The remaining 12 "same" pairs were generated from permutations of three particular sets of letters; these sets were $\mathrm{ABC}, \mathrm{ACR}, \mathrm{BIF}$. The last set may be used to demonstrate the four kinds of permutations that were used. BIF is a trigram that is easily pronounced as a unit although it is relatively low in meaningfulness. FIB is high in both meaningfulness and pronounceability. FBI is high in meaningfulness, but it cannot be pronounced as a single unit. Finally, $\mathrm{BFI}$ is low in both meaningfulness and pronounceability.

The "different" pairs were like the "same" pairs in that both elements were at the same level, either high or low, on meaningfulness and pronounceability (e.g., ABM vs IBM). The elements of the "different" pairs differed from one another in only one letter position.

\section{Results}

The experimental design was essentially factorial with high and low levels of meaningfulness combined with high and low levels of pronounceability. Mean RTs to the four kinds of stimuli are presented in Table 6. An analysis of variance indicated that the effect of pronounceability was significant, $F(1,9)=10.89, \quad p<.01$, as was the interaction between pronounceability and meaningfulness, $F(1,9)=8.25, p<.025$. However, as in the Gibson experiments, meaningfulness was clearly insignificant. A statistical analysis of some selected contrasts indicates that the contrast between high and low pronounceability at a low level of meaningfulness (e.g., BIF vs BFI) accounted for about $90 \%$ of the between-conditions variance.

\section{Discussion}

Calling the key factor in this experiment "pronounceability" is not sufficiently analytic and may actually be misleading. Gibson, Shurcliff, and Yonas (in press) replicated one of the earlier pronounceability studies, but this time with deaf Ss. These Ss showed the same advantage in recognizing pronounceable combinations as hearing Ss did. Thus, pronounceability may not be the proper name for whatever factor it is that we are dealing with. ${ }^{3}$ Gibson (1970) has suggested that the important difference between what have been called pronounceable and unpronounceable items is that the
Table 6

Mean Reaction Times (Msec), Experiment 6

\begin{tabular}{lrrr}
\hline \multirow{2}{*}{$\begin{array}{l}\text { Meaning- } \\
\text { fulness }\end{array}$} & Low & High & $\overline{\mathrm{X}}$ \\
\cline { 2 - 3 } & Pronounceability \\
\hline Low & 986 & 818 & 902 \\
High & 866 & 858 & 862 \\
$\overline{\mathrm{X}}$ & 926 & 838 & 882 \\
\hline
\end{tabular}

pronounceable stimuli follow the well-established rules of English orthography, whereas the unpronounceable ones do not. Such rules would specify, for example, what consonant clusters a word can begin with. This argument explains how deaf $S s$ were able to benefit from pronounceability. This argument also seems to make the difference between items that do or do not follow the rules of orthography very closely related to a familiarity difference. However, familiarity in this case is not to be computed simply by summing bigram or trigram frequencies within a letter string. What must be computed are essentially conditional frequencies for letter combinations in specified positions within a word.

\section{Familiarity}

\section{GENERAL DISCUSSION}

The present results stand in direct contrast to the results of previous experiments that used a perceptual comparison task, since those studies reported no effect of familiarity on perception. However, this discrepancy cannot be considered surprising in view of the procedural details of those experiments as presented in the Introduction. The present conclusion also stands in contrast to other recent investigations which have led to the conclusion that familiarity may influence memory or response bias but not perception (e.g., Smith, 1967; Broadbent, 1967).

Is the present research an anomaly, perhaps the result of faulty equipment or even $\mathrm{E}$ bias? This now seems unlikely. Two as-yet-unpublished reports confirm the finding that familiar items can be compared more quickly than unfamiliar items (Eichelman, 1968; A. Yonas, personal communication). In addition, there are two other recent studies, using quite a different task, that provide very strong evidence of familiarity affecting perception (Reicher, 1969; Wheeler, 1970). Reicher tested the recognition accuracy of the individual letters of a word with a forced-choice procedure. The stimuli were presented tachistoscopically, and the position of the letter to be guessed was made known to the $S$ by means of a poststimulus cue. The results showed that recognition of a letter was more accurate when it was part of a word than when it was part of a meaningless quadrigram. 
Wheeler's study eliminated some possible artifactual causes of Reicher's results and led to the same conclusions. These were excellent, well-controlled studies; it does not look like familiarity effects can be dismissed as "nonperceptual" any longer.

Two directions for further research now seem evident. First, a more detailed analysis of the construct of familiarity is needed. Clearly, letter rotation and rated meaningfulness are very different kinds of manipulations, and it would be surprising if their perceptual effects were identical. Perhaps there are several kinds of familiarities, each having a distinguishable effect on perception. Second, more analytic methods should be used to find the locus (or loci) of the effects of familiarity. To simply say that there are perceptual effects is to stop short of the most interesting aspects of the investigation. Perhaps a stage analysis, as described by Sternberg (1969), would be a fruitful approach to the localization problem.

\section{Judgments of Sameness and Difference}

Surely the most surprising aspect of the present research was the repeated finding that the familiarity effect obtained for "same" judgments but not for "different" judgments. Reaction-time data for "same" and "different" judgments have frequently puzzled psychologists. What has been perplexing is that "same" judgments have sometimes (although not always) been shown to be significantly faster than "different" judgments. This finding is especially troublesome for investigators who think in terms of perceptual models based on feature analysis. On such a model a correct "same" judgment must be the result of an exhaustive comparison of all of the relevant features of the pair of stimuli. However, a "different" judgment might be based on a self-terminating comparison process, stopping as soon as a difference is detected in any feature. But even if the matching process were not self-terminating. an unembellished feature testing model would not lead to a prediction of longer times for "different" judgments than for "same" judgments.

Of course, it is possible to produce elaborations of the basic nodel that fit the data better. For example, a compare-and-check model in which the "same" judgment is the result of one stage of processing and the "different" judgment of two stages has sometimes been proposed (Tversky, 1969). In the first, "compare" stage, the stimuli may be compared by template matching or exhausitve feature lesting. If the comparison indicates identity, then a "same" response is initiated. However, if the items are not identical, the stimulus pair is rechecked, perhaps to discover where the difference lies. This checking process would, in all likelihood, be self-terminating. The present data rule out even this modified model. Clearly, if "same" responses are affected by familiarity, then the first stage must be a locus of the familiarity effect. However, this model has the second stage begin upon termination of the first. Thus, "different" RTs also include the duration of the first stage, and so should also reflect familiarity effects.

A more radical solution to the problem is simply to stop trying to make a single kind of process account for both "same" and "different" judgments. For example, Nickerson (1969) has proposed a model in which there is a "counter" for accumulating difference information and a "clock" for keeping time. The decision rule is to respond "different" if the count criterion is exceeded before the time criterion, otherwise respond "same." The two criteria are set in accordance with task parameters such as speed-accuracy instructions. There are two reasons why this model is probably unsuited to deal with the present data. First, it was designed explicitly for the case in which stimuli vary along a single dimension, such as pitch. Second, Nickerson felt that it was unlikely that the model would be appropriate for stimuli that had readily accessible verbal labels. These reservations seem to be fully warranted. since the model leads to the prediction that where error rates are low (as in all of the present RT experiments). correct "different" RTs will tend to be shorter than correct "same" RTs. However, in Experiments 1 and 2. in which some Ss made no errors at all, precisely the opposite result obtained.

Bamber (1969) has proposed another two-process model. His experiment was designed 10 test some theoretical predictions pertaining to the mode of processing of "features" of complex displays (viz. whether features are processed scquentially or simultaneously). His displays consisted of pairs of letter strings, presented one after the other. On each trial the $S$ had to indicate whether the two strings were the same or different. The two main independent variables were the length of the strings (but on any particular trial the number of letters in the first string was the same as the number in the second) and the number of letters that differed between the two strings. As had been pointed out previously (Egeth, 1966), it is possible to obtain evidence about the mode of processing by examining the function relating "different" RT 10 the number of leatures that differ between two stimuli and the function relating "same" RT to the number of relevant features (i.e., string length in Bamber's case). When Bamber analyzed these two functions, he found a marked discrepancy: "Different" judgments were consistent with a serial self-terminating model, but "same" RTs were much too fast to be consistent with such a model. The RT data in conjunction with an analysis of error rates led Bamber to postulate the existence of two separate stimulus-comparison mechanisms. One of these operates serially upon stimulus features; it emits one of two signals, depending on whether the pair is "same" or "different." The other, faster process is called the identity reporter. It signals only if the pair is the same; otherwise no signal is emitted. Thus, a "same" response can be initiated by either comparison process, although the identity reporter, being faster, will usually be the one to initiate the response.

The data from the present series of experiments clearly support the hypothesis that "same" and "different" judgments may be initiated by independent processes. This is evidenced by the repeated finding that "same" responses are affected by familiarity but "different" responses are not. Unfortunately, it is difficult to go beyond this general statement, because the data from Experiments 4 and 5 (trigrams) differ in an interesting and important way from the data of Experiments 1 and 2 (letter pairs) and from Bamber's data. The difference is that where Bamber found "sames" to be consistently faster than "differents," we find this to be true for pairs of single letters, but not for pairs of trigrams. The reason for this discrepancy is not yet clear. Thus, although we have concluded that "same" and "different" judgments are not opposite sides of the same coin, our reasons are quite different from Bamber's.

The present results invite (but do not demand) the speculation that the difference detector is relentlessly featuristic, whereas the sameness detector is responsive to the configuration of the presented form. It seems that only by being responsive to properties of the overall configuration can the sameness detector use one of those properties (i.e., familiarity) to guide the feature analysis that is going on at the same time within the complex process of sameness detection. Such a seemingly backward mode of operation, from whole to part, seems to be demanded also by the Reicher (1968) and Wheeler (1970) studies.

\section{REFERENCES}

ARCHLR, E. J. Re-evaluation of the meaningfulness of all possible CVC trigrams. Psychological Monographs, 1960, 74, No. 10. BAMBER, D. Reaction times and error rates for 
"same" "“different" judgments of multidimensional stimuli. Perception \& Psychophysics, 1969, 6, 169-174.

BROADBENT, D. E. Word-frequency effect and response bias. Psychological Review, 1967, 74, $1-15$.

EGETH, H. E. Parallel versus serial processes in multidimensional stimulus discrimination. Perception \& Psychophysics, 1966, 9, 245-252.

EGETH, H. E., BLECKER, D. L., \& KAMLET, A. S. Verbal interference in a perceptual comparison task. Perception \& Psychophysics, $1969,6,355-356$.

EGETH, H. E., \& SMITH, E. E. On the nature of errors in a choice reaction task. Psychonomic Science, 1967, 8, 345-346.

EICHELMAN, W. $H$. Letters as units of processing in a visual matching task. Unpublished Master's thesis, University of Oregon, 1968.

GIBSON, E. J. The ontogeny of reading. American Psychologist, 1970, 25, 136-143.

GIBSON, E. J., BISHOP, C. H., SCHIFF, W., \& SMITH, J. Comparison of meaningfulness and pronounceability as grouping principles in the perception and retention of verbal material. Journal of Experimental Psychology, 1964, 67, 173-182.

GIBSON, E. J., PICK, A., OSSER, H., \& HAMMOND, M. The role of graphemc-phoneme correspondence in the perception of words. American Journal of Psychology, 1962, 75, 554-570.

GIBSON, E. J., SHURCLIFF, A., \& YONAS, A.
Utilization of spelling patterns by deaf and hearing subjects. In H. Levin and J. P. Williams (Eds.), Basic studies on reading. New York: Harper \& Row, in press.

GOLDIAMOND, $1 ., \&$ HAWKINS, W. F. Vexierversuch: The log relationship between word-frequency and recognition obtained in the absence of stimulus words, Journal of Experimental Psychology, 1958, 56, 457-463.

HOCHBERG, I. In the mind's eye. In R. N. Haber (Ed.), Contemporary theory and research in visual perception. New York: Holt, Rinehart, \& Winston, 1968. Pp. 309-331.

HOWES, D. H., \& SOLOMON, R. L. Visual duration threshold as a function of word probability. Journal of Experimental Psychology, 1951, 41, 401-410.

NEISSER, U. Cognitive psychology. New York: Appleton-Century-Crofts, 1967.

NICKERSON, R. S. "Same"--"different" response times: A model and a preliminary test. Acta Psychologica, 1969, 30, 257-275.

POLLACK, 1., NORMAN, D. A., \& GALANTER, E. An efficient non-parametric analysis of recognition memory. Psychonomic Science, 1964, 1, 327-328.

POSNER, M. 1., \& MITCHELI, R. F. Chronometric analysis of classification. Psychological Review, 1967, 74, 392-409.

REICHER, G. M. Perceptual recognition as a function of meaningfulness of stimulus material. Journal of Experimental Psychology, $1969,81,275.280$.

ROBINSON, J. S., BROWN, L. T., \& HAYES, W. H. Test of the effect of past visual cxperience in perception. Perceptual \& Motor Skills, 1964, 18, 953-956.

SMITH, E. E. Effects of familiarity on stimulus recognition and categorization. Journal of Experimental Psychology, 1967, 74, 324-332.

STERNBERG, S. Memory-scanning: Mental processes revealed by reaction-time experiments, American Scientist, 1969, 57, 421-457.

TVERSKY, B. Pictorial and verbal encoding in a short-term memory task. Perception \& Psychophysics, 1969, 6, 225-233.

UNDERWOOD, B. J. Experimental Psychology. New York: Appleton-Century-Crofts, 1966.

WHEELER, D. D. Processes in word recognition. Cognitive Psychology, 1970, 1, 59-85.

\section{NOTES}

1. Procedural details were not provided in Hochberg's published article. Wc are grateful to him for sending us a more complete description of this research.

2. Hochberg also used such a familiarity manipulation, although it was not discussed in the published report.

3. A thoughtful reviewer has pointed out that many deaf children talk, and therefore pronounce. They just don't hear what they pronounce. So it is possible that the benefit really is due to pronounccability - at least if these Ss were talkers.

(Accepted for publication July 12.1970.) 